\title{
Nash inequality for Diffusion Processes Associated with Dirichlet Distributions *
}

\author{
Feng-Yu Wang ${ }^{\dagger}$ \\ Center for Applied Mathematics, Tianjin University, Tianjin 300072, China \\ Department of Mathematics, Swansea University, Singleton Park, SA2 8PP, UK
}

Weiwei Zhang

School of Mathematical Sciences, Beijing Normal University, Beijing 100875, China

December 17, 2019

\begin{abstract}
For any $N \geq 2$ and $\alpha=\left(\alpha_{1}, \cdots, \alpha_{N+1}\right) \in(0, \infty)^{N+1}$, let $\mu_{\alpha}^{(N)}$ be the Dirichlet distribution with parameter $\alpha$ on the set $\Delta^{(N)}:=\left\{x \in[0,1]^{N}: \sum_{1 \leq i \leq N} x_{i} \leq 1\right\}$. The multivariate Dirichlet diffusion is associated with the Dirichlet form

$$
\mathscr{E}_{\alpha}^{(N)}(f, f):=\sum_{n=1}^{N} \int_{\Delta^{(N)}}\left(1-\sum_{1 \leq i \leq N} x_{i}\right) x_{n}\left(\partial_{n} f\right)^{2}(x) \mu_{\alpha}^{(N)}(\mathrm{d} x)
$$

with Domain $\mathscr{D}\left(\mathscr{E}_{\alpha}^{(N)}\right)$ being the closure of $C^{1}\left(\Delta^{(N)}\right)$. We prove the Nash inequality

$$
\mu_{\alpha}^{(N)}\left(f^{2}\right) \leq C \mathscr{E}_{\alpha}^{(N)}(f, f)^{\frac{p}{p+1}} \mu_{\alpha}^{(N)}(|f|)^{\frac{2}{p+1}}, \quad f \in \mathscr{D}\left(\mathscr{E}_{\alpha}^{(N)}\right), \mu_{\alpha}^{(N)}(f)=0
$$

for some constant $C>0$ and $p=\left(\alpha_{N+1}-1\right)^{+}+\sum_{i=1}^{N} 1 \vee\left(2 \alpha_{i}\right)$, where the constant $p$ is sharp when $\max _{1 \leq i \leq N} \alpha_{i} \leq \frac{1}{2}$ and $\alpha_{N+1} \geq 1$. This Nash inequality also holds for the corresponding Fleming-Viot process.
\end{abstract}

AMS subject Classification: 60J60, 60H10.

Keywords: Dirichlet distribution, Nash inequality, super Poincaré inequality, diffusion process.

*Supported in part by NNSFC $(11771326,11726627,11831014)$.

${ }^{\dagger}$ Corresponding author: wangfy@tju.edu.cn 


\section{Introduction}

Let $N \geq 1$ be a natural number and $\alpha=\left(\alpha_{1}, \cdots, \alpha_{N+1}\right) \in(0, \infty)^{N+1}$. The Dirichlet distribution $\mu_{\alpha}^{(N)}$ with parameter $\alpha$ is a probability measure on the set

$$
\Delta^{(N)}:=\left\{x=\left(x_{i}\right)_{1 \leq i \leq N} \in[0,1]^{N}:|x|_{1}:=\sum_{i=1}^{N} x_{i} \leq 1\right\}
$$

with density function

$$
\rho(x):=\frac{\Gamma\left(|\alpha|_{1}\right)}{\prod_{1 \leq i \leq N+1} \Gamma\left(\alpha_{i}\right)}\left(1-|x|_{1}\right)^{\alpha_{N+1}-1} \prod_{1 \leq i \leq N} x_{i}^{\alpha_{i}-1}, \quad x=\left(x_{i}\right)_{1 \leq i \leq N} \in \Delta^{(N)},
$$

where $|\alpha|_{1}:=\sum_{i=1}^{N+1} \alpha_{i}$. This distribution arises naturally in Bayesian inference as conjugate prior for categorical distribution, and it describes the distribution of allelic frequencies in population genetics, see for instance [3, 11, 14].

To investigate stochastic dynamics converging to $\mu_{\alpha}^{(N)}$, different models of diffusion processes have been proposed. In this paper, we investigate functional inequalities of these diffusions.

In the following three subsections, we first briefly recall some facts on functional inequalities for Dirichlet forms, as well as known results for diffusion processes associated with the Dirichlet distribution, then propose problems in the direction and state the main result of the paper.

\subsection{Functional inequalities}

In general, let $(\mathscr{E}, \mathscr{D}(\mathscr{E}))$ be a conservative symmetric Dirichlet form on $L^{2}(\mu)$ for some probability space $(E, \mathscr{F}, \mu),(L, \mathscr{D}(L))$ be the associated Dirichlet operator, and $P_{t}:=\mathrm{e}^{t L}, t \geq 0$, be the Markov semigroup. The following is a brief summary from [19] for the Poincaré, log-Sobolev, super Poincaré and Nash inequalities, see also [1,9] and references within.

Firstly, we consider the spectral gap of $L: \operatorname{gap}(L)$ is the largest constant $C>0$ such that the Poincaré inequality

$$
\mu\left(f^{2}\right) \leq \frac{1}{C} \mathscr{E}(f, f), \quad f \in \mathscr{D}(\mathscr{E}), \mu(f)=0
$$

holds. In case this inequality is not available, we say that $L$ does not have spectral gap, and denote $\operatorname{gap}(L)=0$. The Poincaré inequality (1.2) is equivalent to the $L^{2}$-exponential convergence of $P_{t}$ :

$$
\left\|P_{t} f\right\|_{L^{2}(\mu)} \leq \mathrm{e}^{-C t}\|f\|_{L^{2}(\mu)}, \quad t \geq 0, f \in L^{2}(\mu), \mu(f)=0 .
$$

Next, we consider the log-Sobolev constant $C_{L S}(L)$, which is the largest positive constant $C$ such that the log-Sobolev inequality

$$
\mu\left(f^{2} \log f^{2}\right) \leq \frac{2}{C} \mathscr{E}(f, f), \quad f \in \mathscr{D}(\mathscr{E}), \mu\left(f^{2}\right)=1
$$


holds. We have $C_{L S}(L) \leq \operatorname{gap}(L)$. In general, (1.3) implies the exponential decay of $P_{t}$ in entropy:

$$
\mu\left(\left(P_{t} f\right) \log P_{t} f\right) \leq \mathrm{e}^{-C t} \mu(f \log f), \quad t \geq 0, f \in \mathscr{B}^{+}(E), \mu(f)=1,
$$

and in the diffusion setting they are equivalent. Moreover, the log-Sobolev inequality (1.3) holds for some constant $C>0$ if and only if $P_{t}$ is hypercontractive, i.e. $\left\|P_{t}\right\|_{L^{2}(\mu) \rightarrow L^{4}(\mu)}=1$ for large enough $t$.

Finally, we say that $(\mathscr{E}, \mu)$ satisfies the super Poincaré inequality with rate function $\beta:(0, \infty) \rightarrow(0, \infty)$, if

$$
\mu\left(f^{2}\right) \leq r \mathscr{E}(f, f)+\beta(r) \mu(|f|)^{2}, \quad r>0, f \in \mathscr{D}(\mathscr{E}) .
$$

This inequality is equivalent to the uniform integrability of $P_{t}$, i.e. $P_{t}$ has zero tail norm:

$$
\left\|P_{t}\right\|_{t a i l}:=\lim _{R \rightarrow \infty} \sup _{\mu\left(f^{2}\right) \leq 1} \mu\left(\left(P_{t} f\right)^{2} 1_{\left\{\left|P_{t} f\right| \geq R\right\}}\right)=0, \quad t>0 .
$$

When $P_{t}$ has a heat kernel with respect to $\mu$, it is also equivalent to the absence of the essential spectrum of $L$ (i.e. the spectrum of $L$ is purely discrete). The super Poincare inequality generalizes the classical Sobolev/Nash type inequalities. For instance, when $\operatorname{gap}(L)>0$, (1.4) with $\beta(r)=\mathrm{e}^{c\left(1+r^{-1}\right)}$ for some $c>0$ is equivalent to the log-Sobolev inequality (1.3) for some constant $C>0$; while for a constant $p>0$, (1.4) with $\beta(r)=c\left(1+r^{-p}\right)$ holds for some $c>0$ if and only if the Nash inequality

$$
\mu\left(f^{2}\right) \leq C \mathscr{E}(f, f)^{\frac{p}{p+1}} \mu(|f|)^{\frac{2}{p+1}}, \quad f \in \mathscr{D}(\mathscr{E}), \mu(f)=0
$$

holds for some constant $C>0$. They are also equivalent to

$$
\left\|P_{t}-\mu\right\|_{L^{1}(\mu) \rightarrow L^{\infty}(\mu)} \leq \frac{c^{\prime}}{(t \wedge 1)^{p}} \mathrm{e}^{-\operatorname{gap}(L) t}, \quad t>0 .
$$

The later implies the hypercontractivity of $P_{t}$, and hence the log-Sobolev inequality (1.3) for some constant $C>0$.

\subsection{Diffusion processes associated with Dirichlet distributions}

In this part, we recall existing results on functional inequalities for some diffusion processes on $\Delta^{(N)}$, which are reversible with respect to the Dirichlet distribution $\mu_{\alpha}^{(N)}$.

When $N=1$, the Wright-Fisher diffusion on the interval $[0,1]$ generated by

$$
L_{\alpha}^{(1)}:=x(1-x) \partial_{x}^{2}+\left\{\alpha_{1}(1-x)-\alpha_{2} x\right\} \partial_{x} .
$$

The associated Dirichlet form is the closure of $\left(\mathscr{E}_{\alpha}^{(1)}, C^{1}([0,1])\right)$ given by

$$
\mathscr{E}_{\alpha}^{(1)}(f, g)=\int_{0}^{1} x(1-x) f^{\prime}(x) g^{\prime}(x) \mu_{\alpha}^{(1)}(\mathrm{d} x), \quad f, g \in C^{1}([0,1]) .
$$

Due to [15], we have $\operatorname{gap}\left(L_{\alpha}^{(1)}\right)=\alpha_{1}+\alpha_{2}$, and [16, Lemma 2.7] shows that $C_{L S}(L) \geq \frac{\alpha_{1} \wedge \alpha_{2}}{160}$. Moreover, according to $\left[8\right.$, Theorem 2.2], $\left(\mathscr{E}_{\alpha}^{(1)}, \mu_{\alpha}^{(1)}\right)$ satisfies the super Poincaré inequality 
with $\beta(r)=c\left(1+r^{-\left(\frac{1}{2} \vee \alpha_{1} \vee \alpha_{2}\right)}\right)$ for some constant $c>0$, and hence, the Nash inequality holds for $p=\frac{1}{2} \vee \alpha_{1} \vee \alpha_{2}$, which is sharp in the sense that the super Poincaré inequality does not hold if $\lim _{r \rightarrow 0} \beta(r) r^{\frac{1}{2} \vee \alpha_{1} \vee \alpha_{2}}=0$.

When $N \geq 2$, we consider the following three different generalizations of the WrightFisher diffusion arising from population genetics, see e.g. [7, 8, 12, 13, 16].

A. Wright-Fisher diffusion with mutation. Let $|\alpha|_{1}=\sum_{i=1}^{N+1} \alpha_{i}$ and denote $\partial_{i}=$ $\partial_{x_{i}}, 1 \leq i \leq N$. Consider the diffusion process on $\Delta^{(N)}$ generated by

$$
L_{F V}^{\alpha, N}:=\sum_{i, j=1}^{N} x_{i}\left(\delta_{i j}-x_{j}\right) \partial_{i} \partial_{j}+\sum_{i=1}^{N}\left(\alpha_{i}-|\alpha|_{1} x_{i}\right) \partial_{i}
$$

where $\delta_{i j}=1$ if $i=j ;=0$ otherwise. The associated Dirichlet form is the closure of $\left(\mathscr{E}_{F V}^{\alpha, N}, C^{1}\left(\Delta^{(N)}\right)\right)$ given by

$$
\mathscr{E}_{F V}^{\alpha, N}(f, g)=\int_{\Delta^{(N)}} \sum_{i, j=1}^{N} x_{i}\left(\delta_{i j}-x_{j}\right)\left\{\left(\partial_{i} f\right)\left(\partial_{j} g\right)\right\}(x) \mu_{\alpha}^{(N)}(\mathrm{d} x), \quad f, g \in C^{1}\left(\Delta^{(N)}\right)
$$

Again due to $[15,16]$ we have

$$
\operatorname{gap}\left(L_{F V}^{\alpha, N}\right)=|\alpha|_{1}, \quad C_{L S}(L) \geq \frac{1}{160} \min _{1 \leq i \leq N+1} \alpha_{i}
$$

However, the Nash inequality is unknown.

B. GEM process. Let $\beta_{i}=\sum_{j=i+1}^{N+1} \alpha_{j}, 1 \leq i \leq N$. Then $\mu_{\alpha}^{(N)}=\Pi_{\alpha, \beta}$, the GEM distribution with parameter $(\alpha, \beta)$, see e.g. [7]. For $x \in \Delta^{(N)}$ and $1 \leq i, j \leq N$, let

$$
\begin{aligned}
& a_{i j}(x)=x_{i} x_{j} \sum_{k=1}^{i \wedge j} \frac{\left\{\delta_{k i}\left(1-\sum_{1 \leq l \leq k-1} x_{l}\right)-x_{k}\right\} \cdot\left\{\delta_{k j}\left(1-\sum_{1 \leq l \leq k-1} x_{l}\right)-x_{k}\right\}}{x_{k}\left(1-\sum_{1 \leq l \leq k} x_{l}\right)} \\
& b_{i}(x)=x_{i} \sum_{k=1}^{i} \frac{\left\{\delta_{k i}\left(1-\sum_{1 \leq l \leq k-1} x_{l}\right)-x_{k}\right\} \cdot\left\{\alpha_{k}\left(1-\sum_{1 \leq l \leq k-1} x_{l}\right)-\beta_{i} x_{k}\right\}}{x_{k}\left(1-\sum_{1 \leq l \leq k} x_{l}\right)} .
\end{aligned}
$$

The corresponding GEM process introduced in [7] is the diffusion process on $\Delta^{(N)}$ generated by

$$
L_{G E M}^{\alpha, N}:=\sum_{i, j=1}^{N} a_{i j} \partial_{i} \partial_{j}+\sum_{i=1}^{N} b_{i} \partial_{i}
$$

and the associated Dirichlet form is the closure of $\left(\mathscr{E}_{G E M}^{\alpha, N}, C^{1}\left(\Delta^{(N)}\right)\right)$ :

$$
\mathscr{E}_{G E M}^{\alpha, N}(f, g)=\int_{\Delta^{(N)}} \sum_{i, j=1}^{N} a_{i j}(x)\left\{\left(\partial_{i} f\right)\left(\partial_{j} g\right)\right\}(x) \mu_{\alpha}^{(N)}(\mathrm{d} x), \quad f, g \in C^{1}\left(\Delta^{(N)}\right) .
$$


According to [7, Theorem 3.1], we have

$$
\operatorname{gap}\left(L_{G E M}^{\alpha, N}\right)=\alpha_{N}+\alpha_{N+1}, \quad C_{L S}\left(L_{G E M}^{\alpha, N}\right) \geq \frac{1}{160} \min _{1 \leq i \leq N+1} \alpha_{i} .
$$

Moreover, applying [8, (1.4)] for $a_{i}=\alpha_{i}, b_{i}=\beta_{i}:=\sum_{j=i+1}^{N+1} \alpha_{j}$, and using [8, (2.24)], we see that the heat kernel $p_{t}^{G E M}(x, y)$ of the present GEM process with respect to $\mu_{\alpha}^{(N)}$ satisfies

$$
c_{1} t^{-\sum_{i=1}^{N}\left(\frac{1}{2} \vee \alpha_{i} \vee \beta_{i}\right)} \leq \sup _{x, y \in \Delta(N)} p_{t}^{G E M}(x, y) \leq c_{2} t^{-\sum_{i=1}^{N}\left(\frac{1}{2} \vee \alpha_{i} \vee \beta_{i}\right)}, \quad t \in(0,1]
$$

for some constants $c_{2}>c_{1}>0$. So, there exists a constant $C>0$ such that the Nash inequality (1.5) holds for $\left(\mathscr{E}_{G E M} \alpha, N, \mu_{\alpha}^{(N)}\right)$ replacing $(\mathscr{E}, \mu)$ with

$$
p=\sum_{i=1}^{N} \max \left\{\frac{1}{2}, \alpha_{i}, \sum_{i+1 \leq j \leq N+1} \alpha_{j}\right\},
$$

which is sharp in the sense that the Nash inequality fails when this $p$ is replaced by any smaller constant.

C. Multivariate Dirichlet diffusion. This process was introduced in [10], and was used in [2] to describe a fluctuating ensemble of $N$ variables subject to a conservation principle. It can be constructed as the unique solution to the following SDE on $\Delta^{(N)}$ :

$$
\mathrm{d} X_{i}(t)=\left\{\alpha_{i}\left(1-|X(t)|_{1}\right)-\alpha_{N+1} X_{i}(t)\right\} \mathrm{d} t+\sqrt{2\left(1-|X(t)|_{1}\right) X_{i}(t)} \mathrm{d} B_{i}(t), \quad 1 \leq i \leq N
$$

where $B(t):=\left(B_{1}(t), \cdots, B_{N}(t)\right)$ is the $N$-dimensional Brownian motion. The infinitesimal generator of the diffusion is

$$
L_{\alpha}^{(N)}:=\sum_{1 \leq n \leq N}\left(x_{n}\left(1-|x|_{1}\right) \partial_{n}^{2}+\left\{\alpha_{n}\left(1-|x|_{1}\right)-\alpha_{N+1} x_{n}\right\} \partial_{n}\right),
$$

and the associated Dirichelt form is the closure of $\left(\mathscr{E}_{\alpha}^{(N)}, C^{1}\left(\Delta^{(N)}\right)\right)$ :

$$
\mathscr{E}_{\alpha}^{(N)}(f, g)=\int_{\Delta^{(N)}} \sum_{i=1}^{N} x_{i}\left(1-|x|_{1}\right)\left\{\left(\partial_{i} f\right)\left(\partial_{i} g\right)\right\}(x) \mu_{\alpha}^{(N)}(\mathrm{d} x) .
$$

According to [6, Theorem 1.1], we have

$$
\operatorname{gap}\left(L_{\alpha}^{N}\right)=\alpha_{N+1}
$$

Not that when $N=1, \operatorname{gap}\left(L_{\alpha}^{(1)}\right)=\alpha_{1}+\alpha_{2}>\alpha_{2}$.

Moreover, the whole spectrum of $L_{\alpha}^{(N)}$ has been characterized in [6]. In particular, the essential spectrum is empty, so that the super Poincaré inequality

$$
\mu_{\alpha}^{(N)}\left(f^{2}\right) \leq r \mathscr{E}_{\alpha}^{(N)}(f, f)+\beta(r) \mu_{\alpha}^{(N)}(|f|)^{2}, \quad r>0, f \in C^{1}\left(\Delta^{(N)}\right)
$$

holds for some function $\beta:(0, \infty) \rightarrow(0, \infty)$. However, there is no any explicit estimate on $\beta(r)$ and hence, both the log-Soblev and the Nash inequalities are unknown. 


\subsection{Questions and the Main result}

According to the last subsection, the following two things remain unknown.

$\left(Q_{1}\right)$ Nash inequality for the Wright-Fisher diffusion with mutation and multivariate Dirichlet diffusion processes.

$\left(Q_{2}\right)$ Estimate on the log-Sobolev constant for the multivariate Dirichlet diffusion, and the sharp log-Sobolev constant for the Wright-Fisher/Wright-Fisher diffusion with mutation/GEM processes.

In this paper, we only investigate $\left(Q_{1}\right)$, and the main result is the following.

Theorem 1.1. Let $N \geq 2$.

(1) There exists a constant $C>0$ such that the Nash inequality

$$
\mu_{\alpha}^{(N)}\left(f^{2}\right) \leq C \mathscr{E}_{\alpha}^{(N)}(f, f)^{\frac{p}{p+1}} \mu_{\alpha}^{(N)}(|f|)^{\frac{2}{p+1}}, \quad f \in \mathscr{D}\left(\mathscr{E}_{\alpha}^{(N)}\right), \mu_{\alpha}^{(N)}(f)=0
$$

holds for $p=p_{\alpha}:=\sum_{i=1}^{N} 1 \vee\left(2 \alpha_{i}\right)+\left(\alpha_{N+1}-1\right)^{+}$, and the inequality remains true for $\mathscr{E}_{F V}^{(\alpha, N)}$ replacing $\mathscr{E}_{\alpha}^{(N)}$.

(2) If (1.8) holds for some constant $C>0$, then

$$
p \geq \tilde{p}_{\alpha}:=\max \left\{\max _{1 \leq i \leq N+1} \sum_{j \neq i, 1 \leq j \leq N+1} \alpha_{j}, \alpha_{N+1}+\max _{1 \leq i \leq N} \sum_{j \neq i, 1 \leq j \leq N}\left(1 \vee \alpha_{j}\right)\right\} .
$$

(3) If (1.8) with $\mathscr{E}_{F V}^{\alpha, N}$ replacing $\mathscr{E}_{\alpha}^{(N)}$ holds for some constant $C>0$, then

$$
p \geq p_{\alpha}^{\prime}:=\max \left\{\sum_{1 \leq j \leq N} \alpha_{i}, \frac{1}{2} \alpha_{N+1}+\frac{1}{2} \max _{1 \leq i \leq N} \sum_{j \neq i, 1 \leq j \leq N}\left(1 \vee \alpha_{j}\right)\right\} .
$$

Remark 1.2. (1) Let $p_{c}$ be the smallest positive constant $p>0$ such that (1.8) holds for some constant $C>0$, then assertions (1)-(2) in Theorem 1.1 imply $p_{c} \in\left[\tilde{p}_{\alpha}, p_{\alpha}\right]$. In particular, when $\max _{1 \leq i \leq N} \alpha_{i} \leq \frac{1}{2}$ and $\alpha_{N+1} \geq 1$, we have $p_{c}=N+\alpha_{N+1}-1$; that is, in this case the Nash inequality presented in Theorem 1.1(1) is sharp for $\mathscr{E}_{\alpha}^{(N)}$. But the sharpness for $\mathscr{E}_{F V}^{\alpha, N}$ is unknown.

(2) As mentioned in the end of Subsection 1.1 that the Nash inequality (1.8) implies the log-Sobolev inequality

$$
\mu_{\alpha}^{(N)}\left(f^{2} \log f^{2}\right) \leq C \mathscr{E}_{\alpha}^{(N)}(f, f), \quad f \in \mathscr{D}\left(\mathscr{E}_{\alpha}^{(N)}\right), \mu_{\alpha}^{(N)}\left(f^{2}\right)=1
$$

for some constant $C>0$. However, in the moment we do not have any explicit estimate on the log-Sobolev constant $C_{L S}\left(L_{\alpha}^{(N)}\right)$.

(3) Consider the infinite-dimensional setting where $N=\infty$ and $\alpha=\left(\alpha_{i}\right)_{i \in \overline{\mathbb{N}}}$ with $|\alpha|_{1}:=$ $\sum_{i \in \overline{\mathbb{N}}} \alpha_{i}<\infty$. According to $[16,6]$, we have

$$
\operatorname{gap}\left(L_{F V}^{\alpha, \infty}\right)=|\alpha|_{1}, \quad \operatorname{gap}\left(L_{\alpha}^{(\infty)}\right)=\alpha_{\infty} .
$$


Next, [16, Theorem 3.5] shows that the set

$$
D_{0}:=\left\{f \in \mathscr{D}\left(\mathscr{E}_{F V}^{\alpha, \infty}\right): \mu_{\alpha}^{(\infty)}\left(f^{2}\right)+\mathscr{E}_{F V}^{\alpha, \infty}(f, f) \leq 1\right\}
$$

is not uniform integrable in $L^{2}\left(\mu_{\alpha}^{(\infty)}\right)$, so that the super Poincaré inequality is not available for $\left(\mathscr{E}_{F V}^{\alpha, \infty}, \mu_{\alpha}^{(\infty)}\right)$. Indeed, by [18, Theorem 1.2] (see also [17, 19]), if there exists $\beta:(0, \infty) \rightarrow$ $(0, \infty)$ such that

$$
\mu_{\alpha}^{(\infty)}\left(f^{2}\right) \leq r \mathscr{E}_{F V}^{\alpha, \infty}(f, f)+\beta(r) \mu_{\alpha}^{(\infty)}(|f|)^{2}, \quad r>0, f \in \mathscr{D}\left(\mathscr{E}_{F V} \alpha, \infty\right),
$$

then there exists a positive increasing function $F$ on $[0, \infty)$ with $F(r) \uparrow \infty$ as $r \uparrow \infty$ such that

$$
\mu_{\alpha}^{(\infty)}\left(f^{2} F\left(f^{2}\right)\right) \leq \mathscr{E}_{F V} \alpha, \infty(f, f), \quad f \in \mathscr{D}\left(\mathscr{E}_{F V}^{\alpha, \infty}\right), \mu_{\alpha}^{(\infty)}\left(f^{2}\right) \leq 1,
$$

and hence $D_{0}$ is uniform integrable in $L^{2}\left(\mu_{\alpha}^{(\infty)}\right)$. Since $\mathscr{E}_{F V}^{\alpha, \infty} \geq \mathscr{E}_{\alpha}^{(\infty)}$, see the beginning of Section 3 for finite $N$, the super Poincaré inequality is invalid for $\mathscr{E}_{\alpha}^{(\infty)}$.

To prove Theorem 1.1, we will present a localization theorem in Section 2, which enables one to establish the super Poincaré inequality (1.7) by using local inequalities. A complete proof of Theorem 1.1 will be addressed in Section 3 and Section 4.

\section{Preparations}

To establish (1.7) with an explicit rate function $\beta$, the main difficulty comes from the singularity of the density $\rho(x)$ as well as the degeneracy of the diffusion coefficient on the boundary

$$
\partial \Delta^{(N)}=\left\{x=\left(x_{i}\right)_{1 \leq i \leq N} \in \Delta^{(N)}: \min \left\{x_{i}: 1 \leq i \leq N+1\right\}=0\right\}, \quad x_{N+1}:=1-\sum_{i=1}^{N} x_{i} .
$$

To overcome such difficulties, a localization result has been presented in [19, Theorem 3.4.6]. However, this result is less sharp and inconvenient for application to the present model. So, in this section we give a new version of this result. We will also present an additivity property of the super Poincaré inequality, which is more or less trivial but will be used to establish local super Poincaré inequalities in the proof of Theorem 1.1(1).

\subsection{A localization result}

Let $(E, \mathscr{F}, \mu)$ be a separable complete probability space, and $(\mathscr{E}, \mathscr{D}(\mathscr{E}))$ be a conservative symmetric local Dirichlet form on $L^{2}(\mu)$ as the closure of

$$
\mathscr{E}(f, g)=\mu(\Gamma(f, g)), \quad f, g \in \mathscr{D}_{0}(\Gamma)
$$

where $\Gamma: \mathscr{D}(\Gamma) \times \mathscr{D}(\Gamma) \rightarrow \mathscr{B}(E)$ is a positive definite symmetric bilinear mapping, $\mathscr{B}(E)$ is the set of all $\mu$-a.e. finite measurable real functions on $E, \mathscr{D}(\Gamma)$ is a sub-algebra of $\mathscr{B}(E)$, and $\mathscr{D}_{0}(\Gamma):=\left\{f \in \mathscr{D}(\Gamma): f^{2}, \Gamma(f, f) \in L^{1}(\mu)\right\}$ such that 
(a) $\mathscr{D}_{0}(\Gamma)$ is dense in $L^{2}(\mu)$.

(b) $\mathscr{D}(\Gamma)$ is closed under combinations with $\psi \in C([-\infty, \infty])$ such that $\psi$ is $C^{1}$ in $\mathbb{R}$ and $\psi^{\prime}$ has compact support, and $\Gamma(\psi \circ f, g)=\psi^{\prime}(f) \Gamma(f, g) \mu$-a.e. for $f, g \in \mathscr{D}(\Gamma)$.

(c) $\Gamma(f g, h)=g \Gamma(f, h)+f \Gamma(g, h) \mu$-a.e. for $f, g, h \in \mathscr{D}(\Gamma)$.

We aim to establish the super Poincaré inequality (1.4) with an explicit $\beta:(0, \infty) \rightarrow(0, \infty)$.

Theorem 2.1. Let $\phi \in \mathscr{D}(\Gamma)$ be an unbounded nonnegative function such that

$$
h(s):=\sup _{D_{s}} \Gamma(\phi, \phi)<\infty, \quad D_{s}:=\{\phi \leq s\}, \quad s \geq 0,
$$

where $\sup _{\emptyset}=0$ by convention. If there exists $s_{0} \geq 1$ such that for every $s \geq s_{0}$, the local super Poincaré inequality

$$
\mu\left(f^{2}\right) \leq r \mathscr{E}(f, f)+\beta_{s}(r) \mu(|f|)^{2}, \quad r>0, f \in \mathscr{D}(\mathscr{E}),\left.f\right|_{D_{s}^{c}}=0
$$

holds for some decreasing function $\beta_{s}:(0, \infty) \rightarrow(0, \infty)$, and for $s \geq s_{0}$

$$
0<\lambda(s):=\inf \left\{\mathscr{E}(f, f): \mu\left(f^{2}\right)=1,\left.f\right|_{D_{s}}=0\right\} \uparrow \infty \text { as } s \uparrow \infty .
$$

Then

$$
s_{r}:=\inf \left\{s \geq s_{0}: \lambda(s) \geq 8 r^{-1}\right\} \in(0, \infty), \quad r>0,
$$

and there exists a constant $c>0$ such that the super Poincaré inequality (1.4) holds for

$$
\beta(r):=c+\left(2+\frac{r h\left(2 s_{r}\right)}{s_{r}^{2}}\right) \beta_{3 s_{r}}\left(\frac{r}{8+2 r h\left(2 s_{r}\right) s_{r}^{-2}}\right), \quad r>0 .
$$

Proof. By condition (a), it suffices to consider $f \in \mathscr{D}_{0}(\Gamma)$. For any $s \geq s_{0}$ and small $\varepsilon \in(0,1)$, let $\varphi_{i} \in C^{1}([0, \infty])$ with $0 \leq \varphi_{i} \leq 1,\left|\varphi_{i}^{\prime}(s)\right| \leq(1+\varepsilon) s^{-1}, i=1,2$ such that

$$
\left.\varphi_{1}\right|_{[0, s]}=0,\left.\quad \varphi_{1}\right|_{[2 s, \infty]}=1 ;\left.\quad \varphi_{2}\right|_{[0,2 s]}=1,\left.\quad \varphi_{2}\right|_{[3 s, \infty]}=0 .
$$

Let $f_{i}=f \cdot \varphi_{i} \circ \phi, 1 \leq i \leq 2$. Then $f^{2} \leq f_{1}^{2}+f_{2}^{2}$ and by conditions (b) and (c),

$$
\begin{aligned}
\Gamma\left(f_{1}, f_{1}\right) & \leq 2 \Gamma(f, f)+2(1+\varepsilon)^{2} f^{2} s^{-2} 1_{\{\phi \leq 2 s\}} h(2 s) \\
& \leq 2 \Gamma(f, f)+\frac{2(1+\varepsilon)^{2} h(2 s)}{s^{2}} f_{2}^{2}, \\
\Gamma\left(f_{2}, f_{2}\right) & \leq 2 \Gamma(f, f)+\frac{2}{s^{2}}(1+\varepsilon)^{2} f^{2} .
\end{aligned}
$$

In particular, $f_{1}, f_{2} \in \mathscr{D}_{0}(\Gamma) \subset \mathscr{D}(\mathscr{E})$. Combining these with (2.2) and (2.3), we obtain

$$
\begin{aligned}
\mu\left(f^{2}\right) \leq \mu\left(f_{1}^{2}\right)+\mu\left(f_{2}^{2}\right) \leq \frac{2}{\lambda(s)} \mathscr{E}(f, f)+\left(1+\frac{2(1+\varepsilon)^{2} h(2 s)}{s^{2} \lambda(s)}\right) \mu\left(f_{2}^{2}\right) \\
(2.6) \leq\left\{\frac{2}{\lambda(s)}+2 t\left(1+\frac{2(1+\varepsilon)^{2} h(2 s)}{s^{2} \lambda(s)}\right)\right\} \mathscr{E}(f, f)+\frac{2(1+\varepsilon)^{2} t}{s^{2}}\left(1+\frac{2(1+\varepsilon)^{2} h(2 s)}{s^{2} \lambda(s)}\right) \mu\left(f^{2}\right) \\
+\left(1+\frac{2(1+\varepsilon)^{2} h(2 s)}{s^{2} \lambda(s)}\right) \beta_{3 s}(t) \mu(|f|)^{2}, \quad t>0 .
\end{aligned}
$$


Let $r \in(0,1]$, and $s_{r}$ be in $(2.4)$. We have $\lambda\left(s_{r}\right) \geq 8 r^{-1}$, so that

$$
\frac{2}{\lambda\left(s_{r}\right)} \leq \frac{r}{4}, \quad t_{r}:=\frac{r}{8+16 h\left(2 s_{r}\right) /\left[s_{r}^{2} \lambda\left(s_{r}\right)\right]} \geq \frac{r}{8+2 r s_{r}^{-2} h\left(2 s_{r}\right)}
$$

and when $\varepsilon>0$ is small enough,

$$
\begin{aligned}
& (1+\varepsilon)^{2} \leq 2, \quad \frac{2(1+\varepsilon)^{2} h\left(2 s_{r}\right)}{s_{r}^{2} \lambda\left(s_{r}\right)} \leq \frac{r h\left(2 s_{r}\right)}{2 s_{r}^{2}} \\
& 2(1+\varepsilon)^{2} t_{r}\left(1+\frac{2(1+\varepsilon)^{2} h\left(2 s_{r}\right)}{s_{r}^{2} \lambda\left(s_{r}\right)}\right) \leq \frac{3 r}{8} \\
& \frac{2(1+\varepsilon)^{2} t_{r}}{s_{r}^{2}}\left(1+\frac{2(1+\varepsilon)^{2} h\left(2 s_{r}\right)}{s_{r}^{2} \lambda\left(s_{r}\right)}\right) \leq \frac{3 r}{8} \leq \frac{3}{8}, \quad r \in(0,1] .
\end{aligned}
$$

Combining these with (2.6) we arrive at

$$
\mu\left(f^{2}\right) \leq \frac{5 r}{8} \mathscr{E}(f, f)+\frac{3}{8} \mu\left(f^{2}\right)+\left(1+\frac{r h\left(2 s_{r}\right)}{2 s_{r}^{2}}\right) \beta_{3 s}\left(\frac{r}{8+2 r s_{r}^{-2} h\left(2 s_{r}\right)}\right) \mu(|f|)^{2}, \quad r \in(0,1] .
$$

Therefore,

$$
\mu\left(f^{2}\right) \leq r \mathscr{E}(f, f)+\left(2+\frac{r h\left(2 s_{r}\right)}{s_{r}^{2}}\right) \beta_{3 s}\left(\frac{r}{8+2 r s_{r}^{-2} h\left(2 s_{r}\right)}\right) \mu(|f|)^{2}, \quad r \in(0,1] .
$$

Since for the super Poincaré inequality we may take decreasing $\beta$, this finishes the proof.

\subsection{Additivity of super Poincaré inequality}

For every $1 \leq i \leq N$, let $\left(\mathscr{E}_{i}, \mathscr{D}\left(\mathscr{E}_{i}\right)\right)$ be a symmetric Dirichlet form on $L^{2}\left(\mu_{i}\right)$ over a $\sigma$-finite measure space $\left(E_{i}, \mathscr{F}_{i}, \mu_{i}\right)$. Let $\mu=\prod_{i=1}^{N} \mu_{i}$, and $\mathscr{D}(\mathscr{E})$ be the class of $f \in L^{2}(\mu)$ such that for any $1 \leq i \leq N$ and $\left(\prod_{j \neq i} \mu_{j}\right)$-a.e. $x$, we have $f(x, \cdot) \in \mathscr{D}\left(\mathscr{E}_{i}\right)$ and

$$
\mathscr{E}(f, f):=\sum_{i=1}^{N} \int_{\prod_{j \neq i} E_{j}} \mathscr{E}_{i}(f(x, \cdot), f(x, \cdot))\left(\prod_{j \neq i} \mu_{j}\right)(\mathrm{d} x)<\infty .
$$

Consider the following Dirichlet form on $L^{2}(\mu)$ :

$$
\mathscr{E}(f, g):=\sum_{i=1}^{N} \int_{\prod_{j \neq i} E_{j}} \mathscr{E}_{i}(f(x, \cdot), g(x, \cdot))\left(\prod_{j \neq i} \mu_{j}\right)(\mathrm{d} x), \quad f, g \in \mathscr{D}(\mathscr{E})
$$

The following additivity property is a simple consequence of the equivalence between the heat kernel upper bound and the super Poincaré inequality. 
Proposition 2.2. Let $\left\{p_{i}\right\}_{1 \leq i \leq N} \subset(0, \infty)$ such that for any $1 \leq i \leq N$, the super Poincaré inequality

$$
\mu_{i}\left(f^{2}\right) \leq r \mathscr{E}_{i}(f, f)+c_{i}\left(1+r^{-p_{i}}\right) \mu_{i}(|f|)^{2}, \quad r>0, f \in \mathscr{D}\left(\mathscr{E}_{i}\right)
$$

holds for some constant $c_{i}>0$. Then there exists a constant $c>0$ such that

$$
\mu\left(f^{2}\right) \leq r \mathscr{E}(f, f)+c\left(1+r^{-\sum_{i=1}^{N} p_{i}}\right) \mu(|f|)^{2}, \quad r>0, f \in \mathscr{D}(\mathscr{E}) .
$$

Proof. Let $P_{t}^{i}$ be the (sub) Markov semigroup associated with $\left(\mathscr{E}_{i}, \mathscr{D}\left(\mathscr{E}_{i}\right)\right)$. By [19, Theorem 3.3.15(2)], (2.7) implies that $P_{t}^{i}$ has a density $p_{t}^{i}\left(x_{i}, y_{i}\right)$ with respect to $\mu_{i}$ such that

$$
\operatorname{ess}_{\mu_{i} \times \mu_{i}} \sup p_{t}^{i} \leq C_{i}\left(1+t^{-p_{i}}\right), \quad t>0
$$

holds for some constant $C_{i}>0$. Then the semigroup $P_{t}$ associated with $(\mathscr{E}, \mathscr{D}(\mathscr{E}))$ has the density

$$
p_{t}(x, y):=\prod_{i=1}^{N} p_{t}^{i}\left(x_{i}, y_{i}\right), \quad x=\left(x_{1}, \cdots, x_{N}\right), y=\left(y_{1}, \cdots, y_{N}\right) \in E:=\prod_{i=1}^{N} E_{i}
$$

with respect to $\mu$, and

$$
\operatorname{ess}_{\mu \times \mu} \sup p_{t} \leq C\left(1+t^{-\sum_{i=1}^{N} p_{i}}\right), \quad t>0
$$

holds for some constant $C>0$. By [19, Theorem 3.3.15(2)] again, this implies that (2.8) holds for some constant $c>0$.

\section{Proof of Theorem 1.1(1)}

We first observe that for any $f \in C^{1}\left(\Delta^{(N)}\right)$,

$$
\begin{aligned}
& \sum_{i, j=1}^{N} x_{i}\left(\delta_{i j}-x_{j}\right)\left(\partial_{i} f\right) \partial_{j} f=\sum_{i=1}^{N} x_{i}\left(\partial_{i} f\right)^{2}-\sum_{i, j=1}^{N} x_{i} x_{j}\left(\partial_{i} f\right) \partial_{j} f \\
& \geq \sum_{i=1}^{N} x_{i}\left(\partial_{i} f\right)^{2}-\sum_{i, j=1}^{N} x_{i} x_{j} \cdot \frac{\left(\partial_{i} f\right)^{2}+\left(\partial_{j} f\right)^{2}}{2}=\sum_{i=1}^{N} x_{i}\left(1-|x|_{1}\right)\left(\partial_{i} f\right)^{2}
\end{aligned}
$$

So, $\mathscr{E}_{\alpha}^{(N)}(f, f) \leq \mathscr{E}_{F V} \alpha, N(f, f)$, and we only need to prove the desired Nash inequality for $\left(\mathscr{E}_{\alpha}^{(N)}, \mu_{\alpha}^{(N)}\right)$. To this end, it suffices to prove

$$
\mu_{\alpha}^{(N)}\left(f^{2}\right) \leq r \mathscr{E}_{\alpha}^{(N)}(f, f)+c r^{-p_{\alpha}} \mu_{\alpha}^{(N)}(|f|)^{2}, \quad r \in\left(0, r_{1}\right], f \in C^{1}\left(\Delta^{(N)}\right)
$$

for some constants $c, r_{1}>0$. Indeed, this inequality is equivalent to

$$
\mu_{\alpha}^{(N)}\left(f^{2}\right) \leq r \mathscr{E}_{\alpha}^{(N)}(f, f)+c\left(r \wedge r_{1}\right)^{-p_{\alpha}} \mu_{\alpha}^{(N)}(|f|)^{2}, \quad r>0, f \in C^{1}\left(\Delta^{(N)}\right)
$$


Since by [6, Theorem 1.1] the generator $L_{\alpha}^{(N)}$ has spectral gap $\alpha_{N+1}>0$, there holds

$$
\mu_{\alpha}^{(N)}\left(f^{2}\right) \leq \frac{1}{\alpha_{N+1}} \mathscr{E}_{\alpha}^{(N)}(f, f), \quad r>0, f \in C^{1}\left(\Delta^{(N)}\right), \mu_{\alpha}^{(N)}(f)=0 .
$$

Noting that for some constant $c\left(r_{1}, \alpha_{N+1}\right)>0$ we have

$$
\left(r \wedge r_{1}\right)^{-p_{\alpha}} \leq c\left(r_{1}, \alpha_{N+1}\right) r^{-p_{\alpha}}, \quad r \in\left(0, \alpha_{N+1}^{-1}\right)
$$

so that (3.2) and (3.3) yield

$$
\mu_{\alpha}^{(N)}\left(f^{2}\right) \leq r \mathscr{E}_{\alpha}^{(N)}(f, f)+c^{\prime} r^{-p_{\alpha}} \mu_{\alpha}^{(N)}(|f|)^{2}, \quad r>0, f \in C^{1}\left(\Delta^{(N)}\right), \mu_{\alpha}^{(N)}(f)=0
$$

for some constant $c^{\prime}>0$. Minimizing the upper bound in $r>0$, we prove (1.8) for some constant $C>0$ and $p=p_{\alpha}$.

To prove (3.1) using Theorem 2.1, we denote $x_{N+1}=1-|x|_{1}=1-\sum_{i=1}^{N} x_{i}$ and take

$$
\phi(x)=x_{N+1}^{-1}, \quad x=\left(x_{i}\right)_{1 \leq i \leq N} \in \Delta^{(N)} .
$$

Then

$$
D_{s}:=\{\phi \leq s\}=\left\{x \in \Delta^{(N)}: x_{N+1} \geq s^{-1}\right\}, \quad s>1
$$

holds. For the present model we have

$$
\Gamma(\phi, \phi)(x)=\sum_{i=1}^{N} x_{i} x_{N+1}\left(\partial_{i} \phi\right)^{2}(x)=\frac{1-x_{N+1}}{x_{N+1}^{3}} \leq s^{3}, \quad x \in D_{s}, s>0
$$

so that

$$
h(s):=\sup _{D_{s}} \Gamma(\phi, \phi) \leq s^{3}, \quad s>0 .
$$

To apply Theorem 2.1, we take

$$
\mathscr{D}(\Gamma)=\left\{f \in C\left(\Delta^{(N)} ;[-\infty, \infty]\right): f \text { is finite and } C^{1} \text { in } \Delta^{(N)} \backslash\left\{x_{N+1}=0\right\}\right\},
$$

and let

$$
\Gamma(f, g)(x)=1_{\left\{x_{N+1}>0\right\}} \sum_{i=1}^{N} x_{i} x_{N+1}\left(\partial_{i} f\right)(x)\left(\partial_{i} g\right)(x), \quad f, g \in \mathscr{D}(\Gamma) .
$$

Obviously, conditions (a)-(c) hold, and the function $\phi$ in (3.4) meets the requirement of Theorem 2.1. In the following two subsections, we estimate $\lambda(s)$ and $\beta_{s}$ respectively. 


\subsection{Estimate on $\lambda(s)$}

Let $\lambda(s)=\inf \left\{\mathscr{E}_{\alpha}^{(N)}(f, f): f \in C^{1}\left(\Delta^{(N)}\right), \mu_{\alpha}^{(N)}\left(f^{2}\right)=1,\left.f\right|_{D_{s}}=0\right\}$. We will adopt the following Cheeger type estimate for $\lambda(s)$. Let

$$
\begin{gathered}
\partial D_{s}=\left\{x \in \Delta^{(N)}: x_{N+1}=s^{-1}\right\} ; \\
D_{s}^{c}=\Delta^{(N)} \backslash D_{s}, s \geq 1 .
\end{gathered}
$$

Lemma 3.1. If there exists a function $\psi \in C^{2}\left(\Delta^{(N)} \backslash\left\{x_{N+1}=0\right\}\right)$ such that

$$
\Gamma(\psi, \psi)(x):=\sum_{i=1}^{N} x_{i} x_{N+1}\left(\partial_{i} \psi\right)^{2}(x) \leq a_{1}, \quad\left|L_{\alpha}^{(N)} \psi\right|(x) \geq a_{2}, \quad x \in D_{s}^{c}
$$

holds for some constants $a_{1}, a_{2}>0$, and that

$$
\lim _{r \rightarrow \infty} \sup _{x \in \partial D_{r}} x_{N+1}^{\alpha_{N+1}} \sum_{i=1}^{N} x_{i}\left|\partial_{i} \psi(x)\right|=0
$$

then

$$
\lambda(s) \geq \frac{a_{2}^{2}}{4 a_{1}}
$$

Proof. By (3.7), since $D_{s}^{c}$ is connected and $L_{\alpha}^{(N)} \psi$ is continuous, we may assume that $\left.L_{\alpha}^{(N)} \psi\right|_{D_{s}^{c}} \geq a_{2}$, otherwise simply use $-\psi$ replacing $\psi$. Let $\sigma(x)=\operatorname{diag}\left\{\sqrt{x_{i} x_{N+1}}\right\}_{1 \leq i \leq N}$. For any nonnegative $f \in C^{1}\left(\Delta^{(N)}\right)$ with $\left.f\right|_{D_{s}}=0$, we have $\left.f\right|_{\partial D_{s}}=0$. So, by integration by parts formula,

$$
\begin{aligned}
& a_{2} \mu_{\alpha}^{(N)}(f) \leq \mu_{\alpha}^{(N)}\left(f L_{\alpha}^{(N)} \psi\right)=\lim _{r \rightarrow \infty} \int_{D_{r} \backslash D_{s}}\left(\rho f L_{\alpha}^{(N)} \psi\right)(x) \mathrm{d} x \\
& \leq-\mu_{\alpha}^{(N)}(\langle\sigma \nabla f, \sigma \nabla \psi\rangle)+\|f\|_{\infty} \limsup _{r \rightarrow \infty} \int_{\partial D_{r}} \sum_{i=1}^{N} x_{i} x_{N+1} \rho(x)\left|\partial_{i} \psi\right|(x) \mathrm{d} A,
\end{aligned}
$$

where $A$ is the area measure on $\partial D_{r}$ induced by the Lebesgue measure. We have

$$
\partial D_{r}=\left\{x \in \Delta^{(N)}: \sum_{i=1}^{N} x_{i}=1-r^{-1}\right\}, \quad r \geq 2,
$$

and

$$
\int_{\partial D_{r}} \prod_{i=1}^{N} x_{i}^{\alpha_{i}-1} \mathrm{~d} A=\left(1-r^{-1}\right)^{\sum_{i=1}^{N} \alpha_{i}} \int_{\Delta^{(N-1)}}\left(1-\sum_{1 \leq i \leq N-1} x_{i}\right)^{\alpha_{N}-1} \prod_{i=1}^{N-1} x_{i}^{\alpha_{i}-1} \mathrm{~d} x
$$

is bounded in $r \geq 2$. Combining this with (1.1), (3.7), (3.8) and (3.9), we obtain

$$
a_{2} \mu_{\alpha}^{(N)}(f) \leq\left|\mu_{\alpha}^{(N)}(\langle\sigma \nabla f, \sigma \nabla \psi\rangle)\right| \leq \sqrt{a_{1}} \mu_{\alpha}^{(N)}(|\sigma \nabla f|) .
$$


Therefore, for any $f \in C^{1}\left(\Delta^{(N)}\right)$ with $\left.f\right|_{D_{s}}=0$,

$$
\mu_{\alpha}^{(N)}\left(f^{2}\right) \leq \frac{\sqrt{a_{1}}}{a_{2}} \mu_{\alpha}^{(N)}\left(\left|\sigma \nabla f^{2}\right|\right) \leq \frac{2 \sqrt{a_{1}}}{a_{2}} \sqrt{\mu_{\alpha}^{(N)}\left(f^{2}\right) \mu_{\alpha}^{(N)}\left(|\sigma \nabla f|^{2}\right)}
$$

Noting that $\mu_{\alpha}^{(N)}\left(|\sigma \nabla f|^{2}\right)=\mathscr{E}_{\alpha}^{(N)}(f, f)$, we arrive at

$$
\mu_{\alpha}^{(N)}\left(f^{2}\right) \leq \frac{4 a_{1}}{a_{2}^{2}} \mathscr{E}_{\alpha}^{(N)}(f, f), \quad f \in C_{b}^{1}\left(\Delta^{(N)}\right),\left.f\right|_{D_{s}}=0
$$

which finishes the proof.

Lemma 3.2. There exist constants $s_{0}, c_{0}>0$ such that

$$
\lambda(s) \geq c_{0} s, \quad s \geq s_{0} .
$$

Proof. Let $\gamma \in\left[\frac{1}{2}, 1\right) \cap\left(1-\alpha_{N+1}, 1\right)$. Take

$$
\psi(x)=x_{N+1}^{\gamma}, \quad x \in \Delta^{(N)} .
$$

Then

$$
\begin{aligned}
\Gamma(\psi, \psi)(x) & =\sum_{i=1}^{N} x_{i} x_{N+1}\left(\partial_{i} \psi\right)^{2}(x)=\gamma^{2}\left(1-x_{N+1}\right) x_{N+1}^{2 \gamma-1} \\
& \leq \gamma^{2} s^{1-2 \gamma}, \quad x \in D_{s}^{c}
\end{aligned}
$$

and

$$
\lim _{s \rightarrow \infty} \sup _{x \in D_{s}} x_{N+1}^{\alpha_{N+1}} \sum_{i=1}^{N} x_{i}\left|\partial_{i} \psi(x)\right| \leq \lim _{s \rightarrow \infty} \gamma s^{1-\alpha_{N+1}-\gamma}=0 .
$$

Let $s_{0} \geq 1$ such that

$$
\left(1+\alpha_{N+1}-\gamma\right)\left(1-s^{-1}\right) \geq 1-\gamma+s^{-1} \sum_{i=1}^{N} \alpha_{i}, \quad s \geq s_{0} .
$$

Then for $x \in D_{s}^{c}$ and $s \geq s_{0}$,

$$
\begin{aligned}
L_{\alpha}^{(N)} \psi(x) & =\sum_{i=1}^{N}\left\{x_{i} x_{N+1} \partial_{i}^{2} \psi(x)+\left(\alpha_{i} x_{N+1}-\alpha_{N+1} x_{i}\right) \partial_{i} \psi(x)\right. \\
& =\gamma\left(1+\alpha_{N+1}-\gamma\right)\left(1-x_{N+1}\right) x_{N+1}^{\gamma-1}-\gamma x_{N+1}^{\gamma} \sum_{i=1}^{N} \alpha_{i} \\
& \geq \gamma(1-\gamma) s^{1-\gamma} .
\end{aligned}
$$

Combining this with (3.10) and (3.11), we derive from Lemma 3.1 that

$$
\lambda(s) \geq \frac{\gamma^{2}(1-\gamma)^{2} s^{2(1-\gamma)}}{4 \gamma^{2} s^{1-2 \gamma}}=\frac{(1-\gamma)^{2}}{4} s, \quad s \geq s_{0} .
$$




\subsection{Estimate on $\beta_{s}(r)$}

We first present a sharp super Poincaré inequality for a product probability measure, then estimate $\beta_{s}(r)$ using a perturbation argument. Consider the following probability measures on $[0,1]$ :

$$
\mu_{i}(\mathrm{~d} s)=\alpha_{i} s^{\alpha_{i}-1} \mathrm{~d} s, \quad 1 \leq i \leq N
$$

and let $\mu=\prod_{i=1}^{N} \mu_{i}$ on $[0,1]^{N}$. We have the following result.

Lemma 3.3. Let $p(\alpha)=\sum_{i=1}^{N}\left(\frac{1}{2} \vee \alpha_{i}\right)$. There exists a constant $c>0$ such that

$$
\mu\left(f^{2}\right) \leq r \int_{[0,1]^{N}} \sum_{i=1}^{N} x_{i}\left(\partial_{i} f\right)^{2}(x) \mu(\mathrm{d} x)+c\left(r^{-p(\alpha)}+1\right) \mu(|f|)^{2}, \quad r>0, f \in C^{1}\left([0,1]^{N}\right) .
$$

Proof. By Proposition 2.2, it suffices to prove that for every $1 \leq i \leq N$ there exists a constant $c_{i}>0$ such that

$$
\mu_{i}\left(f^{2}\right) \leq r \int_{0}^{1} s f^{\prime}(s)^{2} \mathrm{~d} s+c_{i}\left(1+r^{-\left(\frac{1}{2} \vee \alpha_{i}\right)}\right) \mu_{i}(|f|)^{2}, \quad r>0, f \in C^{1}([0,1]) .
$$

For fixed $1 \leq i \leq N$, we will prove this inequality using isoperimetric constants

$$
\kappa(r):=\inf _{I \subset[0,1], 0<\mu(I) \leq r} \frac{A_{i}((\partial I) \backslash\{0,1\})}{\mu_{i}(I)}, \quad r \in(0,1 / 2)
$$

where $A_{i}$ is the boundary measure induced by $\mu_{i}$ and the intrinsic metric of the square field $\Gamma_{0}(f, f)(s):=s\left(f^{\prime}\right)^{2}(s)$ on $[0,1]$. Let $r \in\left(0, \frac{1}{2}\right)$, for any measurable set $I \subset[0,1]$ with $\mu_{i}(I)=r$, we may find out $a \in(\partial I) \backslash\{0,1\}$ such that $a \geq r^{\frac{1}{\alpha_{i}}}$. Otherwise, $\left[r^{\frac{1}{\alpha_{i}}}, 1\right)$ is either in the interior of $I$ or in that of $I^{c}$. For the first case we have

$$
r=\mu_{i}(I) \geq \alpha_{i} \int_{r^{\frac{1}{\alpha_{i}}}}^{1} s^{\alpha_{i}-1} \mathrm{~d} s=1-r>\frac{1}{2}>r
$$

which is a contraction; while in the second case we may find a small constant $\varepsilon>0$ such that $\left[r^{\frac{1}{\alpha_{i}}}-\varepsilon, 1\right) \subset I^{c}$, so that

$$
r=\mu_{i}(I) \leq \alpha_{i} \int_{0}^{r^{\frac{1}{\alpha_{i}}}-\varepsilon} s^{\alpha_{i}-1} \mathrm{~d} s=\left(r^{\frac{1}{\alpha_{i}}}-\varepsilon\right)^{\alpha_{i}}<r
$$

which is again impossible. Since the intrinsic metric induced by $\Gamma_{0}$ is

$$
\mathrm{d}(s, t):=2|\sqrt{s}-\sqrt{t}|, \quad s, t \in[0,1]
$$

the corresponding boundary measure of $\{a\}$ is given by

$$
A_{i}(\{a\}):=\lim _{\varepsilon \downarrow 0} \frac{\mu_{i}([a-\varepsilon, a])}{2(\sqrt{a}-\sqrt{a-\varepsilon})}=\sqrt{a} a^{\alpha_{i}-1}=a^{\alpha_{i}-\frac{1}{2}} .
$$


Therefore,

$$
\frac{A_{i}((\partial I) \backslash\{0,1\})}{\mu_{i}(I)} \geq \frac{A_{i}(\{a\})}{r} \geq r^{-\left(1 \wedge \frac{1}{2 \alpha_{i}}\right)}
$$

Hence,

$$
\kappa(r) \geq r^{-\left(1 \wedge \frac{1}{2 \alpha_{i}}\right)}, \quad r \in(0,1 / 2) .
$$

According to [19, Theorem 3.4.16(1)], this implies (3.12) for some constant $c_{i}>0$.

Lemma 3.4. Let $p(\alpha)=\sum_{i=1}^{N}\left(\frac{1}{2} \vee \alpha_{i}\right)$. There exist constants $c_{0}, s_{0}>0$ such that for any $s \geq s_{0}$,

$$
\mu_{\alpha}^{(N)}\left(f^{2}\right) \leq r \mathscr{E}_{\alpha}^{(N)}(f, f)+\beta_{s}(r) \mu_{\alpha}^{(N)}(|f|)^{2}, \quad r>0, f \in C^{1}\left(\Delta^{(N)}\right),\left.f\right|_{D_{s}^{c}}=0
$$

holds for

$$
\beta_{s}(r)=c_{0} s^{p(\alpha)+\left(\alpha_{N+1}-1\right)^{+}}\left(r^{-p(\alpha)}+s^{p(\alpha)}\right), \quad r>0 .
$$

Proof. Let $f \in C^{1}\left(\Delta^{(N)}\right),\left.f\right|_{D_{s}^{c}}=0$. For simplicity, we will regard $x_{i}$ as the function mapping $x \in \Delta^{(N)}$ into $x_{i}, 1 \leq i \leq N+1$. Recall that $x_{N+1}:=1-\sum_{i=1}^{N} x_{i}$. Applying Lemma 3.3 to $g:=x_{N+1}^{\left(\alpha_{N+1}-1\right) / 2} f$ replacing $f$, which is supported on $D_{s}$, we may find out constants $c_{1}, c_{2}, c_{3}, c_{4}>0$ such that for any $t>0$ and $s \geq 1$,

$$
\begin{aligned}
& \mu_{\alpha}^{(N)}\left(f^{2}\right)=c_{1} \mu\left(g^{2}\right) \leq c_{1} t \mu_{\alpha}^{(N)}\left(\sum_{i=1}^{N} x_{i}\left(\partial_{i} g\right)^{2}\right)+c_{2}\left(1+t^{-p(\alpha)}\right) \mu_{\alpha}^{(N)}(|g|)^{2} \\
& \leq t c_{3} \mu_{\alpha}^{(N)}\left(\sum_{i=1}^{N} x_{i}\left\{\left(\partial_{i} f\right)^{2}+x_{N+1}^{-2} f^{2}\right\}\right)+c_{2}\left(1+t^{-p(\alpha)}\right) \mu_{\alpha}^{(N)}\left(x_{N+1}^{-\frac{\alpha_{N+1}-1}{2}}|f|\right)^{2} \\
& \leq c_{3} t s \mu_{\alpha}^{(N)}\left(\sum_{i=1}^{N} x_{i} x_{N+1}\left(\partial_{i} f\right)^{2}\right)+c_{3} t s^{2} \mu_{\alpha}^{(N)}\left(f^{2}\right)+c_{2}\left(1+t^{-p(\alpha)}\right) s^{\left(\alpha_{N+1}-1\right)^{+}} \mu_{\alpha}^{(N)}(|f|)^{2} \\
& \leq c_{3} t s \mathscr{E}_{\alpha}^{(N)}(f, f)+c_{3} t s^{2} \mu_{\alpha}^{(N)}\left(f^{2}\right)+c_{2}\left(1+t^{-p(\alpha)}\right) s^{\left(\alpha_{N+1}-1\right)^{+}} \mu_{\alpha}^{(N)}(|f|)^{2} .
\end{aligned}
$$

For any $r>0$, take

$$
t=\frac{r}{2 c_{3} s} \wedge \frac{1}{2 c_{3} s^{2}} .
$$

We may find out a constant $c>0$ such that the above gives

$$
\mu_{\alpha}^{(N)}\left(f^{2}\right) \leq r \mathscr{E}_{\alpha}^{(N)}(f, f)+c\left(r^{-p(\alpha)}+s^{p(\alpha)}\right) s^{p(\alpha)+\left(\alpha_{N+1}-1\right)^{+}} \mu_{\alpha}^{(N)}(|f|)^{2}, \quad r>0 .
$$

Therefore, the proof is finished. 


\subsection{Proof of (3.1)}

By (2.4) and Lemma 3.2, there exist constants $r_{0}, c_{1}>0$ such that

$$
s_{r} \leq c_{1} r^{-1}, \quad r \in\left(0, r_{0}\right]
$$

Combining this with (3.6), we obtain

$$
\frac{r h\left(2 s_{r}\right)}{s_{r}^{2}} \leq 8 r s_{r} \leq 8 c_{1} .
$$

So, there exist constants $r_{1} \in\left(0, r_{0}\right]$ and $c_{2}>1$ such that for any $r \in\left(0, r_{1}\right]$,

$$
\frac{4 s_{r}^{2}+h\left(2 s_{r}\right) r}{2 s_{r}^{2}} \leq c_{2}, \quad \frac{r}{8+2 r h\left(2 s_{r}\right) s_{r}^{-2}} \geq \frac{r}{c_{2}} .
$$

Combining these with (3.13) and (3.14), we may find out constants $c_{3}, c_{4}>0$ such that $\beta(r)$ in (2.5) satisfies

$$
\begin{aligned}
\beta(r) & \leq c+2 \beta_{3 s_{r}}\left(r / c_{2}\right) \leq c_{3} s_{r}^{p(\alpha)+\left(\alpha_{N+1}-1\right)^{+}}\left(r^{-p(\alpha)}+s_{r}^{p(\alpha)}\right) \\
& \leq c+c_{4} r^{-\left\{2 p(\alpha)+\left(\alpha_{N+1}-1\right)^{+}\right\}}, \quad r \in\left(0, r_{1}\right] .
\end{aligned}
$$

This completes the proof since

$$
2 p(\alpha)+\left(\alpha_{N+1}-1\right)^{+}=\sum_{i=1}^{N} 1 \vee\left(2 \alpha_{i}\right)+\left(\alpha_{N+1}-1\right)^{+}=p_{\alpha}
$$

\section{Proof of Theorem 1.1(2)-(3)}

Proof of Theorem 1.1(2). Let (1.8) hold. We aim to prove $p \geq \tilde{p}_{\alpha}^{(1)}$ and $p \geq \tilde{p}_{\alpha}^{(2)}$ respectively, where

$$
\begin{aligned}
\tilde{p}_{\alpha}^{(1)} & :=\alpha_{N+1}+\max _{1 \leq i \leq N} \sum_{1 \leq j \leq N, j \neq i}\left(1 \vee \alpha_{j}\right), \\
\tilde{p}_{\alpha}^{(2)} & :=\max _{1 \leq i \leq N+1} \sum_{1 \leq j \leq N+1, j \neq i} \alpha_{j} .
\end{aligned}
$$

(a) Let $1 \leq i_{0} \leq N$ be such that $\alpha_{i_{0}}=\min _{1 \leq i \leq N} \alpha_{i}$. Let

$$
I_{1}=\left\{i_{0}\right\} \cup\left\{1 \leq i \leq N: \alpha_{i} \leq 1\right\}, \quad I_{2}=\{1, \cdots, N\} \backslash I_{1} .
$$

We have $n_{1}:=\# I_{1} \geq 1, \# I_{2}=N-n_{1}$, and

$$
\sum_{i \in I_{2}}\left(\alpha_{i}-1\right)=\max _{1 \leq i \leq N} \sum_{1 \leq j \leq N, j \neq i}\left(\alpha_{j}-1\right)^{+}=\max _{1 \leq i \leq N} \sum_{1 \leq j \leq N, j \neq i}\left(1 \vee \alpha_{j}\right)+1-N
$$


Take $h \in C^{\infty}(\mathbb{R})$ such that $0 \leq h \leq 1,\left|h^{\prime}\right| \leq 2$ and

$$
\left.h\right|_{(-\infty, 1]}=\left.h\right|_{[4, \infty)}=0,\left.\quad h\right|_{[2,3]}=1 .
$$

Let $\varepsilon_{N}=\frac{1}{32 N^{2}}$ and take

$$
f_{\varepsilon}(x)=\left(\prod_{i \in I_{1}} h\left(\frac{n_{1}^{-1}-x_{i}}{4 N \varepsilon}\right)\right) \cdot \prod_{i \in I_{2}}\left(1-\frac{x_{i}}{2 \varepsilon}\right)^{+}, \quad x \in \Delta^{(N)}, \varepsilon \in\left(0, \varepsilon_{N}\right] .
$$

It is easy to see that $A_{\varepsilon}:=\operatorname{supp} f_{\varepsilon}$ satisfies

$$
\begin{aligned}
A_{\varepsilon}^{(1)} & :=\left[n_{1}^{-1}-12 N \varepsilon, n_{1}^{-1}-8 N \varepsilon\right]^{I_{1}} \times[\varepsilon, 2 \varepsilon]^{I_{2}} \subset A_{\varepsilon} \\
& \subset A_{\varepsilon}^{(2)}:=\left[n_{1}^{-1}-16 N \varepsilon, n_{1}^{-1}-4 N \varepsilon\right]^{I_{1}} \times[0,2 \varepsilon]^{I_{2}} .
\end{aligned}
$$

So, for $x \in A_{\varepsilon}$ we have

$$
\begin{aligned}
2 N \varepsilon & \leq 1-\sum_{i \in I_{1}}\left(n_{1}^{-1}-4 N \varepsilon\right)-2 \varepsilon\left(N-n_{1}\right) \\
& \leq 1-\sum_{i=1}^{N} x_{i}=x_{N+1} \leq 1-\sum_{i \in I_{1}}\left(n_{1}^{-1}-16 N \varepsilon\right) \leq 16 N^{2} \varepsilon,
\end{aligned}
$$

and there exist constants $c_{2}>c_{1}>0$ such that

$$
\begin{aligned}
& c_{1} 1_{A_{\varepsilon}^{(1)}}(x) \varepsilon^{\sum_{i \in I_{2}}\left(\alpha_{i}-1\right)+\alpha_{N+1}-1} \leq\left(1_{A_{\varepsilon}} \rho\right)(x) \leq c_{2} 1_{A_{\varepsilon}^{(2)}}(x) \varepsilon^{\sum_{i \in I_{2}}\left(\alpha_{i}-1\right)+\alpha_{N+1}-1}, \\
& 1_{A_{\varepsilon}^{(1)}}(x) \leq f_{\varepsilon}(x) \leq 1_{A_{\varepsilon}^{(2)}}(x), \\
& \sum_{i=1}^{N} x_{i} x_{N+1}\left(\partial_{i} f_{\varepsilon}\right)^{2}(x) \leq c_{2} \varepsilon^{-1} 1_{A_{\varepsilon}^{(2)}}(x), \quad x \in \Delta^{(N)}, \varepsilon \in\left(0, \varepsilon_{N}\right] .
\end{aligned}
$$

Combining these together we may find out constants $c_{3}, c_{4}>0$ such that for any $\varepsilon \in\left(0, \varepsilon_{N}\right]$,

$$
\begin{aligned}
& \mu_{\alpha}^{(N)}\left(f_{\varepsilon}^{2}\right) \geq \mu_{\alpha}^{(N)}\left(A_{\varepsilon}^{(1)}\right)=\int_{A_{\varepsilon}^{(1)}} \rho(x) \mathrm{d} x \geq c_{3} \varepsilon^{\sum_{i \in I_{2}}\left(\alpha_{i}-1\right)+N+\alpha_{N+1}-1}, \\
& \mu_{\alpha}^{(N)}\left(f_{\varepsilon}\right)^{2} \leq \mu_{\alpha}^{(N)}\left(A_{\varepsilon}^{(2)}\right)^{2} \leq c_{4} \varepsilon^{2 N+2\left(\alpha_{N+1}-1\right)+2 \sum_{i \in I_{2}}\left(\alpha_{i}-1\right)}, \\
& \mu_{\alpha}^{(N)}\left(\sum_{i=1}^{N} x_{i} x_{N+1}\left(\partial_{i} f_{\varepsilon}\right)^{2}\right) \leq c_{2} \varepsilon^{-1} \mu_{\alpha}^{(N)}\left(A_{\varepsilon}^{(2)}\right) \leq c_{4} \varepsilon^{\sum_{i \in I_{2}}\left(\alpha_{i}-1\right)+N+\alpha_{N+1}-2} .
\end{aligned}
$$

Therefore, if (1.7) holds then

$$
\begin{aligned}
& c_{3} \varepsilon^{\sum_{i \in I_{2}}\left(\alpha_{i}-1\right)+N+\alpha_{N+1}-1} \\
& \leq r c_{4} \varepsilon^{\sum_{i \in I_{2}}\left(\alpha_{i}-1\right)+N+\alpha_{N+1}-2}+c_{4} \beta(r) \varepsilon^{2 N+2\left(\alpha_{N+1}-1\right)+2 \sum_{i \in I_{2}}\left(\alpha_{i}-1\right)}, \quad r>0, \varepsilon \in\left(0, \varepsilon_{N}\right] .
\end{aligned}
$$

This is equivalent to

$$
1-\frac{c_{4}}{c_{3}} r \varepsilon^{-1} \leq \frac{c_{4}}{c_{3}} \beta(r) \varepsilon^{\sum_{i \in I_{2}}\left(\alpha_{i}-1\right)+N+\alpha_{N+1}-1}, \quad r>0, \varepsilon \in\left(0, \varepsilon_{N}\right] .
$$


Let $r_{N}=\frac{c_{3}}{2 c_{4}} \varepsilon_{N}$. For any $r \in\left(0, r_{N}\right]$, we take $\varepsilon=\frac{2 c_{4}}{c_{3}} r \in\left(0, \varepsilon_{N}\right]$ in the above inequality to derive from (4.1) that

$$
\beta(r) \geq \frac{c_{3}}{2 c_{4}} \varepsilon^{1-\sum_{i \in I_{2}}\left(\alpha_{i}-1\right)-N-\alpha_{N+1}}=c r^{-\tilde{p}_{\alpha}^{(1)}}, \quad r \in\left(0, r_{N}\right]
$$

for some constant $c>0$. Since (1.8) implies (1.7) for $\beta(r)=c\left(1+r^{-p}\right)$ for some constant $c>0$, this implies $p \geq \tilde{p}_{\alpha}^{(1)}$.

(b) On the other hand, $1 \leq i_{0} \leq N+1$ be such that $\alpha_{i_{0}}=\min _{1 \leq i \leq N+1} \alpha_{i}$. Let

$$
I=\left\{i: i \neq i_{0}, 1 \leq i \leq N+1\right\}
$$

For any $\varepsilon \in(0,1)$, we take

$$
f_{\varepsilon}(x)=\prod_{i \in I}\left(\varepsilon-x_{i}\right)^{+}, \quad x \in \Delta^{(N)}
$$

Then on the support of $f_{\varepsilon}$ we have

$$
x_{i} x_{N+1} \leq \min \left\{x_{i}, x_{N+1}\right\} \leq \varepsilon, \quad 1 \leq i \leq N .
$$

So, as shown in (a) we may find out a constant $a \in(0,1)$ such that for all $\varepsilon \in(0,1)$,

$$
\begin{aligned}
& \mu_{\alpha}^{(N)}\left(f_{\varepsilon}^{2}\right) \geq a \varepsilon^{3 N+\sum_{i \in I}\left(\alpha_{i}-1\right)}=a \varepsilon^{2 N+\sum_{i \in I} \alpha_{i}}, \\
& \mu_{\alpha}^{(N)}\left(f_{\varepsilon}\right)^{2} \leq a^{-1} \varepsilon^{4 N+2 \sum_{i \in I}\left(\alpha_{i}-1\right)}=a^{-1} \varepsilon^{2 N+2 \sum_{i \in I} \alpha_{i}}, \\
& \mu_{\alpha}^{(N)}\left(\left|\sigma \nabla f_{\varepsilon}\right|^{2}\right) \leq a^{-1} \varepsilon^{3 N+\sum_{i \in I}\left(\alpha_{i}-1\right)-1}=a^{-1} \varepsilon^{2 N+\sum_{i \in I} \alpha_{i}-1},
\end{aligned}
$$

and these together with (1.8) imply

$$
p \geq \sum_{i \in I} \alpha_{i}=\max _{1 \leq i \leq N+1} \sum_{j \neq i, 1 \leq j \leq N+1} \alpha_{j}=\tilde{p}_{\alpha}^{(2)} .
$$

Proof of Theorem 1.1(3). Let $f_{\varepsilon}$ be as in (4.2). We have

$$
\mathscr{E}_{F V}^{\alpha, N}\left(f_{\varepsilon}, f_{\varepsilon}\right) \leq \mu_{\alpha}^{(N)}\left(\sum_{i=1}^{N} x_{i}\left(\partial_{i} f_{\varepsilon}\right)^{2}\right) \leq c_{4} \varepsilon^{\sum_{i \in I_{2}}\left(\alpha_{i}-1\right)+N+\alpha_{N+1}-1}
$$

for some constant $c_{4}>0$. Combining this with the first two lines in (4.3), we derive from (1.7) with $\mathscr{E}_{F V}^{\alpha, N}$ replacing $\mathscr{E}_{\alpha}^{(N)}$ that

$$
c_{3} \varepsilon^{\sum_{i \in I_{2}}\left(\alpha_{i}-1\right)+N+\alpha_{N+1}-1} \leq c_{4} \varepsilon^{\sum_{i \in I_{2}}\left(\alpha_{i}-1\right)+N+\alpha_{N+1}-3}+c_{4} \varepsilon^{2 \sum_{i \in I_{2}}\left(\alpha_{i}-1\right)+2 N+2 \alpha_{N+1}-2} \beta(r),
$$

thus,

$$
1-\frac{c_{4} r}{c_{3} \varepsilon^{2}} \leq \frac{c_{4}}{c_{3}} \beta(r) \varepsilon^{\sum_{i \in I_{2}}\left(\alpha_{i}-1\right)+N+\alpha_{N+1}-1}
$$


Taking $\varepsilon=\left(\frac{2 c_{3} r}{c_{4}}\right)^{\frac{1}{2}}$ for small $r>0$ we arrive at

$$
\beta(r) \geq c r^{-\frac{1}{2}\left(N+\alpha_{N+1}-1+\sum_{i \in I_{2}}\left(\alpha_{i}-1\right)\right)} .
$$

Combining this with (1.8) implies $p \geq \frac{1}{2} \alpha_{N+1}+\frac{1}{2} \max _{1 \leq i \leq N} \sum_{j \neq i, 1 \leq j \leq N}\left(1 \vee \alpha_{j}\right)$.

On the other hand, take

$$
f_{\varepsilon}(x)=\prod_{1 \leq i \leq N}\left(\varepsilon-x_{i}\right)^{+}
$$

for small $\varepsilon>0$. Then there exists a constant $a \in(0,1)$ such that for small $\varepsilon>0$ we have

$$
\begin{aligned}
& \mu_{\alpha}^{(N)}\left(f_{\varepsilon}^{2}\right) \geq a \varepsilon^{2 N+\sum_{1 \leq i \leq N} \alpha_{i}} \\
& \mu_{\alpha}^{(N)}\left(f_{\varepsilon}\right)^{2} \leq a^{-1} \varepsilon^{2 N+2 \sum_{1 \leq i \leq N} \alpha_{i}}, \\
& \mathscr{E}_{F V}^{\alpha, N}\left(f_{\varepsilon}, f_{\varepsilon}\right) \leq \mu_{\alpha}^{(N)}\left(\sum_{i=1}^{N} x_{i}\left(\partial_{i} f_{\varepsilon}\right)^{2}\right) \leq a^{-1} \varepsilon^{2 N+\sum_{1 \leq i \leq N} \alpha_{i}-1},
\end{aligned}
$$

so that (1.8) for $\mathscr{E}_{F V}^{\alpha, N}$ implies $p \geq \sum_{1 \leq i \leq N} \alpha_{i}$.

Acknowledgement. The authors would like to than the referee for helpful comments on an earlier version of the paper.

\section{References}

[1] Bakry D, Gentil I, Ledoux M. Analysis and Geometry of Markov Diffusion Operators. Springer, 2014.

[2] Bakosi J, Ristorcelli J R. A stochastic diffusion process for the Dirichlet distribution. Int J Stoch Anal, 2013, Article ID 842981, 7 pages.

[3] Connor R J, Mosimann, J E. Concepts of independence for proportions with a generalization of the Dirichlet distribution. J. Amer Statist Assoc, 1969, 64: 194-206.

[4] Davies E B, Simon B. Ultracontractivity and the heat kernel for Schrödinger operators and Dirichlet Laplacians. J Funct Anal, 1984, 59: 335-395.

[5] Epstein C L, Mazzeo R. Wright-Fisher diffusion in one dimension. SIAM J Math Anal, 2010, 42: 568-608.

[6] Feng S, Miclo L., Wang F.-Y. Poincare inequality for Dirichlet distributions and infinitedimensional generalizations. Lat Amer J Probab Math Stat, 2017, 14: 361-380.

[7] Feng S, WangF.-Y. A class of infinite-dimensional diffusion processes with connection to population genetics. J Appl Probab, 2007, 44: 938-949.

[8] Feng S, WangF.-Y. Harnack inequality and applications for infinite-dimensional GEM processes. Potential Anal, 2016, 44: 137-153. 
[9] Gross L. Logarithmic Sobolev inequalities and contractivity properties of semigroups. In: Dirichlet Forms. Lecture Notes in Math 1563 (Springer, Berlin). 1993, 54-88.

[10] Jacobsen M. Examples of multivariate diffusions: time-reversibility; a Cox-IngersollRoss type process. Department of Theoretical Statistics, Preprint 6, University of Copenhagen. 2001.

[11] Johnson N. L. An approximation to the multinomial distribution, some properties and applications. Biometrika, 1960, 47: 93-102.

[12] Miclo L. About projections of logarithmic Sobolev inequalities. Lecture Notes in Math. 1801 (J. Azéma, M. Émery, M. Ledoux, M. Yor Eds). Springer, 2003, 201-221.

[13] Miclo L. Sur l'inégalité de Sobolev logarithmique des opérateurs de Laguerre à petit paramétre. Lecture Notes in Math. 1801 (J. Azéma, M. Émery, M. Ledoux, M. Yor Eds). Springer, 2003, 222-229.

[14] Mosimann J E. On the compound multinomial distribution, the multivariatedistribution, and correlations among proportions. Biometrika, 1962, 49: 65-82.

[15] Shimakura N. Equations différentielles provenant de la génetique des populations. Tôhoka Math J, 1977, 29: 287-318.

[16] Stannat W. On validity of the log-Sobolev inequality for symmetric Fleming-Viot operators. Ann Probab, 2000, 28: 667-684.

[17] Wang F.-Y. Functional inequalities for empty essential spectrum. J Funct Anal, 2000, 170: $219-245$.

[18] Wang F.-Y. Functional inequalities, semigroup properties and spectrum estimates. Inf Dimens Anal Quant Probab Relat Topics, 2000, 3: 263-295.

[19] Wang F.-Y. Functional Inequalities, Markov Semigroups and Spectral Theory. Science Press, 2005. 